\title{
El desarrollo como estrategia política de China
}

\section{Georgina Higueras'}

En una sociedad socialista, la ley del valor no desempeña un papel regulador en el campo de la producción. La acción reguladora la ejercen las leyes de desarrollo planificado y armónico, así como los planes económicos del Estado. MAO ZEDONG

Resumen: La pandemia ha consolidado a China no solo como fuerza confiable para impulsar la recuperación mundial sino también como actor responsable, preocupado por el futuro de los más desfavorecidos, lo que ha facilitado a Pekín avanzar en su estrategia política de alzarse como representante y voz de los países en vías de desarrollo. Desde su posición de segunda potencia económica y frente al creciente antagonismo de Estados Unidos, la República Popular fomenta un nuevo concepto de desarrollo que califica de "innovador, coordinado, ecológico, abierto e inclusivo". Con este desarrollo no ligado a los valores occidentales de democracia y derechos humanos, pero basado en los principios de soberanía, no injerencia en los asuntos internos y beneficio mutuo, busca priorizar la lucha contra la pobreza en la Agenda 2030 de Naciones Unidas, al tiempo que promueve la Franja y la Ruta para expandir sus relaciones comerciales y tecnológicas.

Palabras clave: Desarrollo, Xi Jinping, Consenso de Pekín, Plan Quinquenal,Agenda 2030, La Franja y la Ruta, EEUU.

\footnotetext{
' Periodista especializada en Asia. Profesora asistente en la Universidad de Hubei (Wuhan, R. P. China) y otras universidades.
} 


\section{Development as a political strategy in China}

Abstract: The pandemic has consolidated China's role as a force that can be relied upon to aid in a worldwide recovery as well as that of a responsible actor who cares about the future of the most vulnerable. From its position as the second-biggest economic power, and faced with growing antagonism from the United States, the Popular Republic is promoting a new concept of development which it describes as "innovative, coordinated, ecological, open and inclusive". This development is not linked to western values of democracy and human rights but based on principles of sovereignty, non-interference with internal affairs and mutual benefit. Itaims to prioritise the fight against poverty in the UN Agenda 2030 while promoting the Belt and Road project to expand its commercial and technological relationships.

Key words: Development, Xi Jinping, Beijing Consensus, Five-year Plan, Agenda 2030, Blt and Road, USA.

\section{Le développement comme stratégie poli- tique en Chine}

Résumé: La pandémie a consolidé le rôle de la Chine en tant que force sur laquelle on peut compter pour aider à la reprise mondiale, ainsi que celui d'un acteur responsable qui se soucie de l'avenir des plus vulnérables. Du haut de sa position de deuxième puissance économique, et face à l'antagonisme croissant des États-Unis, la République Populaire promeut un nouveau concept de développement qu'elle décrit comme «innovant, coordonné, écologique, ouvert et inclusif». Ce développement n'est pas lié aux valeurs occidentales de démocratie et de droits de I'homme mais repose sur les principes de souveraineté, de non-ingérence dans les affaires intérieures et de bénéfice mutuel. II vise à donner la priorité à la lutte contre la pauvreté dans l'Agenda 2030 de l'ONU tout en promouvant le projet Une Ceinture, Une Route pour étendre ses relations commerciales et technologiques.

Mots clé: Développement, Xi Jinping, Consensus de Pekín, Plan Quinquenal, Agenda 2030, Une Ceinture, Une Route, Ceinture et Route, États Unis.

\section{Introducción}

El buen comportamiento de la economía china mientras una significativa parte de Occidente seguía sumido en el COVID19 ha confirmado la tendencia que la convirtió durante la gran crisis de 2008 en una importante y confiable fuerza para impulsar la recuperación de la economía mundial. Su enorme implicación y su firme adhesión al mercado abierto y a la globalización apaciguan el tsunami de estos tiempos borrascosos y juegan un valioso papel. Asida al desarrollo como estrategia política y al beneficio mutuo en su implementación, China busca asentarse como principal actor internacional para, junto con la ONU y otras instituciones multilaterales, priorizar un desarrollo no ligado a los valores occidentales, pero abanderado de la lucha contra la pobreza en la Agenda 2030. 
Cuando se recrudece el enfrentamiento ideológico que lidera el presidente estadounidense, Joe Biden, quien pretende forjar "una alianza de democracias para frenar a la dictadura china", Pekín no se arredra y responde que el sistema liberal "no representa la voluntad de la comunidad internacional" 2, según declaró el pasado marzo el ministro de Relaciones Exteriores Wang Yi. De igual manera, al defender "la prioridad a la subsistencia y al desarrollo económico como condición previa para disfrutar en plenitud de los derechos humanos" ${ }^{3}$, China gana adeptos entre los países más desfavorecidos que se sienten maltratados por Occidente, que les explotó en la era colonial y después les impuso onerosas condiciones para acceder a créditos necesarios para consolidar su independencia económica.

El empeño chino en acabar con la pobreza tiene mucho que ver con su visión del desarrollo que, aunque ha variado desde la fundación de la República Popular en 1949, se ha apoyado siempre en la necesidad de mejorar la vida de la población como método para frenar los levantamientos populares que tan profundamente han marcado la historia del Imperio del Centro. De igual manera, la estrategia del "socialismo con características chinas" hacia los países más desfavorecidos es impulsar un desarrollo que mejore la situación vital de la sociedad sin tener en cuenta el sistema político o la represión existente.

En contraste con el llamado Consenso de Washington, que proponía como receta para el crecimiento económico una serie de principios ligados al liberalismo, el Consenso de Pekín, como lo popularizó Joshua Cooper Ramo, tiene como objetivo fundamental la mejora del nivel de vida de la sociedad como "única manera de evitar el caos" ${ }^{\prime \prime}$ y carece de reglas específicas. Sus recomendaciones son las pautas que han permitido a China convertirse en la segunda potencia económica, entre las que destacan la innovación, el gradualismo, la experimentación constante, la apertura y la autodeterminación, sin bien en especial esta última es casi imposible de aplicar por la mayoría de los países en vías de desarrollo.

Tras haber alcanzado el hito histórico de sacar de la pobreza a 800 millones de personas en menos de medio siglo y haber erradicado la pobreza extrema, Pekín se ve legitimado para promover su nuevo concepto de desarrollo que presenta

${ }^{2}$ S. LEE MYERS (2021).

${ }^{3}$ G. HIGUeRAS (2014).

${ }^{4}$ M. QUIAN Y G. Fremd (2010). 
como "innovador, coordinado, ecológico, abierto e inclusivo", según se refleja en el 'XIV Plan Quinquenal para el Desarrollo Económico y Social Nacional y los Objetivos a Largo Plazo hasta el Año 2035', elaborado por el Comité Central del Partido Comunista de China (PCCh), en octubre de 2020.

En China, la visión del desarrollo de los países está muy ligada a la del suyo propio, de ahí la conveniencia de repasar los planes quinquenales, ya que ofrecen una clara perspectiva de cómo varía de uno a otro el enfoque, además del "objetivo y la orientación del desarrollo a lo largo plazo"b. Mao Zedong, obsesionado por la instauración de una sociedad igualitaria, veía con cierto recelo el endeudamiento y la dependencia de la Unión Soviética que preconizaba la industrialización frente a la autosuficiencia campesina y no era muy partidario de este instrumento, pese a lo cual Zhou Enlai y Chen Yun elaboraron el I Plan Quinquenal (1953-1957), que se centró en la transformación socialista de la agricultura y en la industria pesada. Para Mao, la economía se encontraba al servicio del gran objetivo político que era la construcción del Estado socialista. Hacía hincapié en el "desarrollo de la sociedad por todos y para todos". ${ }^{7}$

Pese al aislamiento internacional que soportó China debido "a la actitud de Estados Unidos de boicotear de manera sistemática la entrada de la República Popular en la $\mathrm{ONU}^{\prime \prime}$ y a la grave situación económica que padecía después de más de un siglo de guerras, el Gobierno comunista inició la ayuda al exterior en 1950, proporcionando asistencia material a la República Popular Democrática de Corea y a Vietnam para apoyar la lucha de Ho Chi Minh contra los franceses. Los inicios de la ayuda exterior están recogidos en el primer libro blanco de cooperación exterior, publicado en 2011.

Hasta el momento, Pekín ha lanzado tres documentos sobre su ayuda exterior. El último, aparecido en enero de 2021 con el título de 'La cooperación internacional de China para el desarrollo en la nueva era', ${ }^{\prime \prime}$ vincula las acciones a las grandes

${ }^{5}$ R. TANG (consultado el 03/05/2021).

6 "Diez planes quinquenales de desarrollo económico y social de la Nueva China", disponible en http:// spanish.china.org.cn/spanish/222422.htm (consultado el 08/05/2021).

${ }^{7}$ C. Hoffman (1971).

${ }^{8}$ R. TAMAmes (2007) (pp. 31-39).

9 "China emite libro blanco sobre cooperación internacional para el desarrollo", Xinhua, disponible 
iniciativas de Xi Jinping: La Franja y la Ruta y la creación de una comunidad global de futuro compartido. El texto destaca la cooperación Sur-Sur e insiste en que China es el mayor país en vías de desarrollo, al contrario de lo que sostiene Estados Unidos, que la considera una potencia y su principal rival.

El paso más importante dado por Pekín en el ordenamiento de la ayuda exterior ha sido la puesta en marcha de la Agencia China de Cooperación Internacional para el Desarrollo (AChCID), aprobada por la Asamblea Popular Nacional (el Parlamento), en la sesión plenaria de marzo de 2018. Uno de sus principales objetivos era "romper el cuello de botella", que suponía la fragmentación en más de 23 ministerios, comisiones y oficinas en la provisión de la ayuda exterior.

Alzándose como la mayor defensora de la cooperación Sur-Sur, China busca "establecer un nuevo modelo de relaciones internacionales basadas en el respeto mutuo, la igualdad, la justicia y la ganancia compartida"10. Se trata de una diplomacia claramente alejada del respeto a los derechos humanos y el imperio de la ley que permea las instituciones internacionales establecidas a instancias de Estados Unidos tras su victoria en la Segunda Guerra Mundial.

\section{El desarrollo en la estrategia del PCCh}

"Terminada la primera fase de la reforma agraria, rota la base económica de la burguesía y concluida la guerra de Corea"11, China entró en una nueva etapa en la que tenía que buscar su modelo de desarrollo. Empezando porque su revolución había sido campesina y no obrera como en la Unión Soviética, las diferencias sociales entre ambos países eran enormes, pero no había más solución que adoptar el modelo soviético de planificación, si la República Popular quería convertirse en una potencia, después de los fracasos que acumuló el régimen nacionalista del Kuomintang en sus intentos de implementar reformas económicas de tipo occidental.

en http://spanish.xinhuanet.com/2021-01/10/c_139655778.htm (consultado el 03/05/2021).

10 "China's International Development Cooperation in the New Era", The State Council Information Office, PRC, 2021, disponible en http://english.scio.gov.cn/whitepapers/2021-01/10/content_77099782_8. $\mathrm{htm}$ (consultado el 10/05/2021).

11 J. D. SPENCE (2011). 
Los planes quinquenales trazan las líneas maestras de actuación y las iniciativas de desarrollo económico y social de los cinco años siguientes. Su objetivo es el crecimiento económico nacional, pero al incluir las directrices de las políticas para las reformas facilitan entender los nuevos conceptos de desarrollo y los cambios en la estrategia a seguir. El diplomático Tang Rui los define como "un buen testimonio del desarrollo progresivo de China hacia un país en proceso de modernización ${ }^{\prime \prime 2}$. Tal vez el más ambicioso de todos ellos sea el 'XIV Plan Quinquenal para el Desarrollo Económico y Social Nacional y los Objetivos a Largo Plazo hasta el Año 2035', aprobado en marzo de 2021 por la APN, que en el mismo enunciado indica que su propósito no es para un lustro sino para tres.

En la década de los 50, la recién creada República Popular carecía de obreros y burócratas, por lo que realizó un reclutamiento masivo de quienes debían acabar con la pobreza del país. "Mao creó una fuerza laboral china sin incentivos materiales ni la promesa de realización individual o familiar"13, con la que puso en marcha el Primer Plan Quinquenal (1953-1957) que, en su búsqueda de la transformación socialista, logró un aumento impresionante de la producción industrial. Excesivamente centrado en la industria pesada, su éxito condujo a la locura del Gran Salto Adelante, que trastocó el segundo plan (1958-1962).

Obsesionado por conseguir un modelo de desarrollo con el que superar en 15 años la producción occidental, el PCCh dejó de prestar la atención necesaria a la agricultura, lo que unido a distintos desastres naturales provocó una terrible hambruna, en la que entre 1958 y 1962 hubo, según el historiador Frank Dikkoter, "45 millones de muertos innecesarios"14. Como consecuencia, el ajuste de las políticas ocasionó un periodo de transición de tres años hasta poner en marcha el tercer plan quinquenal (1966-1970). En realidad, los cinco primeros planes fueron, como la política del PCCh, de experimentación y búsqueda incesante de nuevas directrices para conseguir el modelo de desarrollo deseado.

Hubo que esperar a la toma del poder por Deng Xiaoping para emprender una reforma profunda del sistema económico chino, por lo que el sexto plan (1981-1985) se dedicó principalmente al ajuste y solución de los problemas planteados por los anteriores. La restructuración acometida facilitó que el séptimo plan (1986-1990)

\footnotetext{
${ }^{12}$ R. TANG (consultado el 03/05/2021).

${ }^{13}$ D. M. LAMPTON (2008) (pp. 3-7).

${ }^{14}$ F. DIKÖTER (2017).
} 
alumbrara las líneas generales del nuevo modelo de desarrollo, el denominado "socialismo con características chinas", que combina con notable flexibilidad las acciones del mercado y del Estado.

Desde su llegada en 2002 a la Secretaría General del partido, Hu Jintao se manifestó a favor de introducir en el modelo de desarrollo chino una perspectiva científica, que lo abordara de forma integral y centrado en la persona, con el fin de corregir los desequilibrios generados por más de dos décadas de excesivo hincapié en el crecimiento del Producto Interior Bruto. La "fábrica del mundo", como se comenzó a llamar a China, echaba demasiado humo y los problemas sociales se hacían insostenibles con protestas y manifestaciones por todo el país.

Según el sinólogo de la Universidad de Boston Joseph Fewsmith, el concepto de desarrollo científico es "una suma de desarrollo integral, coordinado y sostenible"15. Esta visión más humanista del desarrollo, que aborda el colapso de los servicios sociales y la creciente brecha entre ricos y pobres, fue incluida en los estatutos del PCCh durante la celebración del XVII Congreso, en noviembre de 2007 y marcó el XI Plan Quinquenal (2006-2010). La teoría, que hacía gala de una gran flexibilidad, proponía también un modelo de desarrollo menos dependiente de la inversión extranjera directa y más atento a la sostenibilidad, evitando el derroche de agua y de recursos energéticos no renovables e impulsando la lucha contra la contaminación medioambiental.

Xi Jinping, que conoce personalmente las miserias de los lugares remotos del país -él vivió en una cueva de la región de Shanxi y transportó estiércol durante la Gran Revolución Cultural-, está obsesionado con que el desarrollo no deje a nadie atrás ni en China ni en el resto del mundo. Su liderazgo autoritario de tinte confuciano hace hincapié en la ciencia y la tecnología como soporte para la "implementación de una estrategia de desarrollo basado en la innovación"16, mientras se afana en construir para 2035 una sociedad modestamente acomodada y en que la República Popular al cumplir su centenario en 2049 se haya convertido en "una nación rica, fuerte, democrática, civilizada, moderna, socialista y en armonía".

Convencido de que el desarrollo es la mejor forma de defender los "intereses legítimos" del pueblo chino, las dos grandes iniciativas del presidente -La Franja y la Ruta y Creado en China 2025-pretenden un desarrollo abierto, cooperativo,

15J. FEWSMITH (consultado el 11/05/2021).

16J. XI (2014) (pp. 149-166). 
pacífico y de beneficio mutuo. "No habrá desarrollo sostenible en el mundo mientras unos países sean cada vez más ricos y otros se hundan cada vez más en la pobreza y el atraso" ${ }^{17}$ repite $\mathrm{Xi}$ en sus discursos.

EI XIV Plan Quinquenal (2021-2025) destaca que China logró eliminar la pobreza absoluta en 2020. El texto, que pone las metas en 2035, incluida la de duplicar para entonces la riqueza de 2020, insiste en que es "el mayor país en vías de desarrollo" y que con su progreso ha contribuido al 30\% del crecimiento económico mundial. Por el contrario, EEUU sostiene que su rival es una potencia con aspiraciones de alcanzar la cúspide económica y tecnológica global y, por tanto, no es un país en desarrollo, sino que se encuentra entre los avanzados. Según estimaciones para 2021 del Fondo Monetario Internacional, China se sitúa por Paridad de Poder Adquisitivo en el puesto 70 del mundo, con 18.931 dólares per cápita, inmediatamente detrás de Tailandia, República Dominicana y México.

La innovación es primordial en la estrategia a largo plazo de este plan, que forma parte del conjunto de iniciativas impulsadas por Xi y apoyadas en las nuevas tecnologías, como el Plan para el Desarrollo de la Industria Robótica, el Plan Trienal de Implementación de la Inteligencia Artificial Internet Plus y el Plan de las Nuevas Infraestructuras, entre otros. Todos ellos tienen como objetivos que China sea "el primer país en el que las infraestructuras estén gestionadas por tecnologías como $5 G$, que sus ciudades sean más sostenibles y menos contaminadas, que para 2030 China sea ni más ni menos que el líder mundial en Inteligencia Artificial (IA), y que en 2035 sea el país que fije globalmente los próximos estándares en ámbitos como IA, $5 \mathrm{G}$ o el Internet de las cosas"18. Todo ello pasa por la creación de un nuevo modelo de desarrollo, denominado circulación dual, que garantice la autonomía del país manteniendo la apertura de su mercado, para promover la cooperación internacional y el beneficio mutuo.

\section{El consenso de Pekín}

El economista británico John Williamson presentó en 1989 el decálogo de medidas económicas y financieras de corte neoliberal que se denominó Consenso de Washington, debido a que tanto el Tesoro estadounidense como las instituciones

\footnotetext{
${ }^{17}$ lbídem (pp. 307-344).

${ }^{18}$ C. F. GonZÁlez (2021) (pp. 19-37).
} 
internacionales con sede en esa ciudad (el Fondo Monetario Internacional, el Banco Mundial y el Banco Interamericano de Desarrollo) respaldaron todas las disposiciones. Orientadas al mercado, su objetivo fue impulsar la reforma económica en América Latina a través de un ajuste estructural, con el fin de mejorar la eficiencia y la competitividad. El decálogo que se impuso a los países incluía: 1) disciplina fiscal, 2) recorte del gasto público; 3) incremento de la base tributaria; 4) liberalización de las tasas de interés; 5) libre flotación del tipo de cambio; 6) liberalización del comercio internacional (eliminación de aranceles y contingentes); 7) liberalización de la inversión extranjera directa; 8) privatización de empresas públicas; 9) desregulación; y 10) garantías de seguridad legal para los derechos de propiedad industrial ${ }^{19}$. Los resultados no fueron los esperados y el tremendo coste que, entre otros, tuvo el fin de los subsidios para la población más pobre fue muy criticado, tanto por los sectores izquierdistas de los países que sufrieron estas medidas, como por economistas progresistas como Stiglitz, Krugman y otros muchos.

El desafío a esas políticas que arruinaron países como Indonesia y Argentina, proviene de lo que Joshua Cooper Ramo llamó en 2004 el Consenso de Pekín, cuyos componentes fundamentales son cinco: (1) 'capitalismo de Estado' (en tanto que el Estado juega un papel primordial en la estrategia y decisiones tanto de las empresas públicas como de las privadas y modela el mercado); (2) gradualidad en las reformas; (3) apertura al exterior, tanto para el comercio internacional como para la inversión extranjera directa; (4) autoritarismo político, y (5) flexibilidad y gran capacidad de adaptación a las circunstancias.

Según Ramo, el modelo chino de crecimiento está ligado a la innovación y la experimentación constantes, utiliza la economía y la gobernanza para promover un desarrollo sostenible y equitativo, está tan orientado al cambio social como al económico y supone un claro compromiso con los países en vías de desarrollo. Añade que, si es importante que los logros en el desarrollo estén cambiando China, mucho más importante es el "gigantesco efecto" que sus ideas están teniendo fuera de sus fronteras, al marcar el paso a numerosos países que quieren desarrollarse.

China está reuniendo recursos para eclipsar a EEUU en muchas áreas esenciales del escenario internacional y para la construcción de un entorno que dificulte la acción hegemónica de Estados Unidos ${ }^{20}$.

${ }^{19}$ S. A. BeRUMEN (consultado el 10/05/2021).

${ }^{20} \mathrm{~J}$. C. RAMO (2004). 
El PCCh aceptó complacido la teoría de Ramo, que difundió ampliamente la diplomacia china con la intención de acrecentar su poder blando. En línea con los principios de la política exterior de la República Popular, el Consenso de Pekín se adhiere a la no injerencia en los asuntos internos y defiende la autodeterminación de los países en vías de desarrollo frente al orden internacional existente y los valores occidentales. Sus objetivos se centran en fomentar el desarrollo incrementando la producción y la construcción de infraestructuras sin tener en cuenta el régimen del país con el que coopera, ni si respeta o no los derechos humanos.

Al igual que el Consenso de Washington, el de Pekín también ha generado mucho debate. Los críticos, como el economista Yang Yao, afirman que el Consenso de Pekín no se ajusta a la realidad china, cuyo éxito económico se alcanzó cuando el régimen se movió "inequívocamente hacia las doctrinas de mercado de la economía neoclásica" ${ }^{21}$, centrada en la oferta y la demanda como fuerzas impulsoras de la producción. Yao señala que en la República Popular impera un modelo autoritario con una fuerte participación estatal en la economía, cuya característica no es la innovación, sino la aplicación de lo inventado por otros e importado para la fabricación e integración de sus empresas como ensambladoras en las grandes redes de distribución globales.

En esta misma línea, Scott Kennedy considera la teoría de Ramo "un mito" y sostiene entre otros puntos que lejos de promover un desarrollo equitativo ha generado una enorme brecha entre ricos y pobres.

La desigualdad, no la igualdad, ha sido un sello distintivo del crecimiento de China. Varios cientos de millones de personas han salido de la pobreza, pero independientemente de cómo se mida (personal, sectorial o regional) la desigualdad ha aumentado ${ }^{22}$.

La crisis de 2008, sin embargo, supuso un antes y un después para la imagen de China en el exterior. Muchos países miraron al milagro económico que se libraba en la República Popular en búsqueda de una solución, aunque en buena medida no han tenido en cuenta que a este han contribuido tanto el tamaño del país como sus instituciones. El economista Enrique Fanjul considera que la excepcionalidad del Imperio del Centro "hace difícilmente exportable" su modelo, en el que juega un papel muy importante la masiva captación de inversiones extranjeras, algo que no es fácil de conseguir. Reconoce, sin embargo, que el Consenso de Pekín se hizo muy atractivo para numerosos países en desarrollo, que fueron los más

${ }^{21}$ Y. YAO (2010).

${ }^{22}$ S. KENNEDY (2010). 
golpeados por la crisis originada en "la codicia de muchos agentes económicos y en la ineficiencia de muchos sistemas de control" ${ }^{23}$ de los países industrializados.

Son muchos los expertos que consideran que "China es un país único", tanto por su historia, como por su evolución y por las características geográficas y sociales del país, por lo que su modelo no es exportable. "Sería inútil que otros países en desarrollo emularan las instituciones y prácticas financieras y legales nacionales de China como una forma de lograr el crecimiento económico" 24, señala Yasheng Huang, profesor estadounidense de Gestión Internacional en el Instituto Tecnológico de Massachussets.

La nueva diplomacia que promueve Xi Jinping destaca que lo importante es "la paz, el desarrollo, la cooperación y el beneficio mutuo" 25 , siempre dentro del respeto a las políticas de cada país. China, tras las críticas que generó su apoyo a la tesis de Ramo, rechazó que esté promoviendo su modelo entre los países en vías de desarrollo e indicó que cada uno debe seguir su propio camino hacia el progreso. Los más desfavorecidos, sin embargo, ven en la estrategia china una luz al final del túnel de su subdesarrollo. Después de décadas de aplicar la amarga medicina que les propusieron las instituciones internacionales creadas por los países ricos y de aceptar la injerencia en sus asuntos internos con la esperanza de que se les abriera una puerta hacia la sociedad del bienestar que publicitan las grandes agencias internacionales, aplauden la política de Pekín que les ofrece sin condiciones la construcción de infraestructuras para su modernización a cambio de las materias primas que China necesita para proseguir su escalada económica.

En Occidente se han alzado las voces que tildan la política china de neocolonialista e incluso neoimperialista. Entre los críticos se afirma que con su defensa del beneficio mutuo y la consigna de ganar-ganar "pretende marcar distancias con las estrategias occidentales y, por extensión, usar el discurso anticolonialista para crear vínculos con África y América Latina" 26 . Pero entre los africanos cunde más el apoyo que la crítica. "El hecho es que África está encontrando nuevas oportuni-

${ }^{23}$ E. FANJUL (2009).

${ }^{24}$ Y. HUANG (2008) (pp. 1-10).

25J. YANG (2015).

${ }^{26}$ J. P. Cardenal y H. Araújo (2011) (p. 118). 
dades para adquirir nuevas tecnologías, capital exterior, capacidades humanas y conocimiento científico de China" ${ }^{27}$. En América Latina hay un mayor recelo a que el desembarco masivo de manufacturas, tecnología y proyectos de infraestructuras a cambio de materias primas provoque una desindustrialización en alguna de las economías más avanzadas, como la brasileña o la chilena, donde las empresas locales se ven forzadas a competir con las grandes compañías chinas respaldadas por el Estado.

Por el contrario, el PCCh destaca que su modelo es una muestra de la generosidad de China al priorizar a países pequeños en sus esfuerzos de cooperación y al representar a los países en desarrollo ante las instituciones internacionales, una actitud semejante al concepto occidental de hegemonía benigna ${ }^{28}$.

La dadivosidad con que sostuvo el Renminbi durante la crisis asiática de 1997/98 le valió un enorme reconocimiento entre su vecindad, donde varios países como Vietnam, Camboya, Laos, Mongolia, Filipinas y Malasia, e incluso en cierta extensión Corea del Norte, replicaron buena parte del modelo chino. Después de la crisis de 2008, varios gobiernos de Asia Central también han facilitado la permeabilización de sus sistemas económicos con la influencia china.

\section{La Agencia China de Cooperación Internacional para el Desarrollo}

El Gobierno chino decidió hace tres años centralizar la ayuda al exterior en un organismo independiente, que informa directamente al Consejo de Estado, aunque bajo la supervisión del Ministerio de Exteriores. La Agencia China de Cooperación Internacional para el Desarrollo (AChCID) se fundó en abril de 2018 con el objetivo de mejorar la gestión y conseguir una mayor coordinación, eficiencia e impacto de los programas de ayuda. Su constitución forma parte de la amplia reforma de la política exterior china que está llevando a cabo el presidente $\mathrm{Xi}$, para darle mayor peso y hacer un uso más efectivo de sus recursos, con el fin de reforzar el interés de los países en sumarse al gran instrumento estratégico de la diplomacia china que es la Franja y la Ruta.

\footnotetext{
${ }^{27}$ T. LUMUMBA-KASONGO (2011).
}

28 J. YANG (2011). 
Cuando el PCCh aceptó la política de reforma y apertura de Deng Xiaoping en diciembre de 1978, la renta per cápita china era inferior a la media de los países africanos. En estas cuatro décadas "el país ha pasado de ser uno de los principales receptores de ayuda exterior a convertirse en un proveedor fundamental de recursos de inversión y desarrollo para el Sur global" 29 . Las cifras hablan por sí solas: entre 1980 y 2000, China recibió 38.000 millones de dólares en ayuda exterior, mientras que entre 2013 y 2018 , su ayuda al desarrollo alcanzó una cifra aproximada a los 7.000 millones de dólares anuales. Además, entre 2000 y 2014 concedió a países en vías de desarrollo unos 350.000 millones de dólares en préstamos sin intereses, préstamos en condiciones favorables y subvenciones. ${ }^{30}$

Tradicionalmente, la ayuda exterior china ha sido bilateral y se concedía bajo los principios de respeto a la soberanía, no interferencia en los asuntos internos e igualdad y beneficio mutuo. Al convertirse en potencia económica, ha querido aproximarse al método de Asistencia Oficial para el Desarrollo (AOD) utilizado por la Organización para la Cooperación y el Desarrollo Económicos (OCDE). En este acercamiento a la OCDE se enmarca la creación de la AChCID, con la que China pretende que la asistencia sea más efectiva, más transparente y más coherente, al tiempo que hace frente a las críticas de opacidad y mercantilización de la ayuda provenientes tanto de gobiernos occidentales como de las grandes ONG internacionales.

Conforme China se ha ido consolidando como potencia han arreciado las críticas a su modelo económico y a los millonarios créditos concedidos a los países pobres ${ }^{31}$ con la supuesta intención de convertirse en el primer proveedor de servicios bancarios del mundo en desarrollo. Pero como dice el economista indio Arvind Subramanian,

a causa de su peculiar estrategia de desarrollo, también será un país comerciante de dimensiones abrumadoras - mucho más grande, con el tiempo, que sus mayores socios-, lo que lo hará menos susceptible a la influencia extranjera. ${ }^{32}$

${ }^{29}$ C. CHENG (2019).

${ }^{30} \mathrm{https}$ ///www.actuemosjuntos.org/post/conoces-la-nueva-agencia-de-cooperaci\%C3\%B3n-internacional-para-el-desarrollo-de-china (consultado el 28/05/2021).

${ }^{31} \mathrm{~J}$. Wheatley, J. COTterlLL Y N. MUNSH (2020).

${ }^{32}$ A. Subramanian (2013) (pp. 304-307).. 
En Occidente, gobiernos, prestamistas multilaterales y ONGs se asocian para brindar asistencia al desarrollo de los países pobres. China, sin embargo, no ve el desarrollo como ayuda. En los años posteriores a Mao, el desarrollo estaba íntimamente ligado al propio impulso nacional para conseguir un alto crecimiento económico y una mejora del nivel de vida. Esta percepción se trasladó a su diplomacia y dio origen "a una amplia gama de compromisos comerciales, a menudo respaldados por el Estado, que van desde el comercio y la inversión hasta los préstamos". ${ }^{33}$

El PCCh trató de impulsar la influencia de la República Popular en el mundo a través del internacionalismo proletario y el apoyo a la descolonización y al desarrollo de los países no alineados. Alentó, además, la llamada "diplomacia de chequera" con la que trataba de atraerse el reconocimiento diplomático en una lucha abierta contra el régimen nacionalista de Chiang Kai-shek refugiado en la isla de Taiwán, que representó a China en las Naciones Unidas hasta 1971. La ayuda al exterior se convirtió así en una pesada carga para el país al "ascender a una media entre 1971 y 1975 del 5,9 por ciento del gasto total del Gobierno, con un máximo del 6,9 por ciento en 1973". ${ }^{34}$ Tras la muerte de Mao en 1976 y el establecimiento de relaciones diplomáticas con EEUU en 1979, se produjo un descenso muy significativo de la asistencia exterior y un cambio en el tipo de proyectos que se apoyaban.

En las cuatro décadas siguientes, la ayuda exterior se impregnó de un cierto enfoque mercantilista, reflejo de su dependencia hasta la fundación de la AChCID, del Ministerio de Comercio y de los dos bancos creados en 1994 -el Export Import Bank of China y el China Development Bank-, que financiaban todos los proyectos de ayuda al desarrollo. Con China posicionada como primer país en desarrollo, el Gobierno destaca que sus esfuerzos de asistencia y apoyo se enmarcan en la cooperación Sur-Sur, en la que la Franja y la Ruta juega un papel muy relevante.

El hecho de que la AChCID esté supervisada por el Ministerio de Exteriores aumenta la capacidad de la diplomacia china de utilizar la ayuda al exterior para incrementar su propia agenda política. Ya desde que las relaciones con Taiwán se deterioraron tras el ascenso a la presidencia en 2016 de Tsai Ing-wen por el independentista Partido Democrático Progresista, Pekín regresó a la práctica de prometer fuertes ayudas a los escasos países, en su mayoría pequeños, que aún mantienen relaciones con Taipei para que las rompan y en su lugar establezcan

${ }^{33}$ M. FERCHEN (2020).

${ }^{34}$ C. CHENG (2019). 
con China. Los tres últimos que han abandonado la "isla rebelde" son El Salvador, las islas Salomón y Kiribati.

Una de las grandes incógnitas aún por resolver es cual será la relación de la AChCID con las ONG chinas y si les abrirá la puerta a la ejecución y entrega de la ayuda. Los proyectos chinos han recibido muchas críticas por "carecer de un toque comunitario", y la promoción de la Franja y la Ruta no ha hecho más que aumentarlas al carecer de vínculos con las personas de las comunidades de los países en los que se realizan los proyectos. Los voluntarios y miembros de las ONG pueden desplegarse con facilidad sobre el terreno y ejercer esa conexión, pero de momento la agencia "ha prestado una atención mínima a trabajar con las ONG"35.

China tampoco tiene mucha experiencia en ayuda de emergencia y ayuda humanitaria en zonas de conflicto, por lo que quiere evaluar sus capacidades y gestionar los riesgos de involucrarse en regiones inestables o en zonas afectadas por conflictos. El enfoque más generalista y menos comercial de la ayuda al exterior que tiene la AChCID puede ser de gran utilidad en este nuevo empeño.

La segunda Conferencia de Alto Nivel de las Naciones Unidas sobre Cooperación Sur-Sur, celebrada en Buenos Aires en marzo de 2019 hizo hincapié en la importancia de la cooperación triangular "para lograr cambios positivos en los países del Sur, en sus esfuerzos por erradicar la pobreza, en todas sus formas y dimensiones" ${ }^{\prime 36}$. Denominada también trilateral es la que involucra a un donante tradicional, uno emergente y un país socio en desarrollo, lo que complementa tanto la cooperación Norte-Sur como la Sur-Sur.

Más allá de la colaboración iniciada en los años ochenta del pasado siglo con diversas agencias de la ONU, China tiene poca experiencia en cooperación triangular. La AChCID puede servir de gran impulso, como revela que nada más crearse participó en dos proyectos en el Pacífico: uno de agua China-Nueva Zelanda-Islas Cook y otro de control de malaria China-Australia-Papúa Nueva Guinea.

Aunque Pekín concede una importancia mucho mayor a la ayuda bilateral, también quiere avanzar en cooperación trilateral por dos motivos: 1) responder a las demandas de los donantes tradicionales de un mayor compromiso a medida que

${ }^{35}$ D. ZHANG y H. J (2021).

36 https://www.un.org/pga/73/es/2019/03/21/second-high-level-united-nations-conference-onsouth-south-cooperation-2/ (consultado el 1/06/2021). 
ellos eliminan gradualmente la ayuda bilateral a China, y 2) mejorar la eficacia de la ayuda china con el aprendizaje de ciertas habilidades y experiencias de los donantes tradicionales y de las agencias de la ONU. ${ }^{37}$

\section{Aportaciones del nuevo libro blanco del desarrollo}

El Gobierno chino publicó en enero de 2021 su tercer libro blanco sobre ayuda exterior, titulado 'Cooperación internacional de China para el desarrollo en la nueva era'. Los dos primeros aparecieron en 2011 y 2014. Según los expertos, el nuevo texto es mucho más que una actualización de los anteriores. "Debe verse como parte del discurso más amplio de China sobre el papel que busca desempeñar en el mundo: es el manifiesto de China para el liderazgo en el desarrollo global" ${ }^{38}$

Como en el caso de la AChCID, el cambio de terminología desde ayuda exterior a cooperación internacional para el desarrollo refleja una tendencia global que busca introducir una mayor igualdad y transparencia entre donante y receptor para lo que es fundamental sustituir el concepto de ayuda por el de cooperación. En cuanto a la nueva era, se refiere a la iniciada en noviembre de 2012 con el ascenso de Xi Jinping a la Secretaría General del PCCh en el XVIII Congreso. De hecho, el libro se refiere ampliamente a las grandes iniciativas mundiales de Xi: La comunidad de destino compartido para la humanidad y la Franja y la Ruta.

Su aparición en mitad de la pandemia no es casual. Dedica un capítulo completo a la ayuda humanitaria y asegura que el esfuerzo realizado en el exterior por China en la lucha contra el Covid19 es "la misión de asistencia humanitaria de emergencia más intensa y de mayor escala" en la historia de la República Popu$\operatorname{lar}^{39}$. Antes de iniciarse la 'diplomacia de las vacunas', en la que se disputa con la Unión Europea el primer puesto de las donaciones, el texto exalta lo que se llamó la 'diplomacia de las mascarillas', que supuso el envío a decenas de países de cientos de millones de estas, además de otros materiales y equipos médicos especializados para luchar contra la pandemia. Además, el texto destaca un apoyo explícito a

${ }^{37}$ D. Zhang y L. TRAJBer Walsbich (2020).

${ }^{38} \mathrm{H}$. ZHANG, "China's manifesto for leadership in global development", disponible en https://pandapawdragonclaw.blog/2021/02/08/chinas-manifesto-for-leadership-in-global-development/ (consultado el 10/05/2021).

${ }^{39}$ D. ZHANG (2021). 
la Organización Mundial de la Salud y el compromiso de suministrar en los dos próximos años 2.000 millones de dólares en vacunas, equipamiento y asistencia médica a los países más pobres y más afectados por el Covid19.

Después de conseguir que su Teoría del desarrollo científico se incluyera en los estatutos del PCCh en el XVII Congreso, celebrado en 2007, Hu Jintao quiso complementarlo con un impulso al diálogo regional y mundial que fomentara la paz, y desde la tesis de que el crecimiento económico de China tiene vocación pacifista. Estas ideas son la base del primer libro blanco, denominado 'Desarrollo pacífico de China', en el que se declaraba que

el desarrollo pacífico constituye la opción estratégica para hacer realidad la modernización, enriquecer al pueblo y aumentar el poder del país y hacer mayores contribuciones a la civilización y el progreso mundiales. ${ }^{00}$

El segundo libro blanco se titula 'Ayuda de China al exterior 2014' y tiene como "objetivo apoyar a los países subdesarrollados a reducir la pobreza y mejorar el nivel de vida de sus pueblos". Aparecido después de lanzar la iniciativa de la Franja y la Ruta, el texto hace hincapié en las subvenciones, créditos sin intereses y préstamos sin condiciones que Pekín brinda a las naciones en vías de desarrollo.

Cuando China concede ayuda al extranjero, el país se adhiere a los principios de no imponer ninguna condición política, no intervenir en los asuntos internos de las naciones receptoras y respetar plenamente su derecho de optar independientemente por su propio camino y modelo de desarrollo ${ }^{41}$,

subraya el documento.

Aunque tanto en este texto como en el publicado en 2021 se palpa la obsesión de Xi Jinping por erradicar la pobreza y su convencimiento de que el primer derecho humano es el de la alimentación, el tercer libro es un documento mucho más elaborado y ambicioso, que pone en valor los logros en el desarrollo de China con el objetivo de que sea vista como líder en gobernanza global. Destaca su papel de gran potencia, con autoridad para hablar en nombre de los países en vías de desarrollo y defender los intereses de todos ellos, en especial de los más vulnerables.

\footnotetext{
40 "Primer Libro Blanco: Desarrollo Pacífico de China", Ministerio de Asuntos Exteriores de la R. P. China, disponible en https://www.fmprc.gov.cn/esp/zxxx/+862206.shtml (consultado el 31/05/2021).

${ }_{41}$ "China publica libro blanco sobre ayuda al extranjero", Diario del Pueblo, 10/07/2014, disponible en http://spanish.peopledaily.com.cn/n/2014/0710/c31621-8753691.html (consultado el 6/06/2021).
} 
Al ayudar a otros países en desarrollo a reducir la pobreza y mejorar la vida de sus habitantes, China trabaja junto con ellos para reducir la brecha Norte-Sur, eliminar el déficit en el desarrollo, establecer un nuevo modelo de relaciones internacionales basado en el respeto mutuo, la equidad, la justicia y la cooperación con ganancias mutuas y para construir un mundo abierto, inclusivo, limpio y hermoso que disfrute de una paz duradera, seguridad universal y prosperidad común ${ }^{42}$,

subraya.

Distribución de la Ayuda Exterior de China por Regiones, 2013-2018

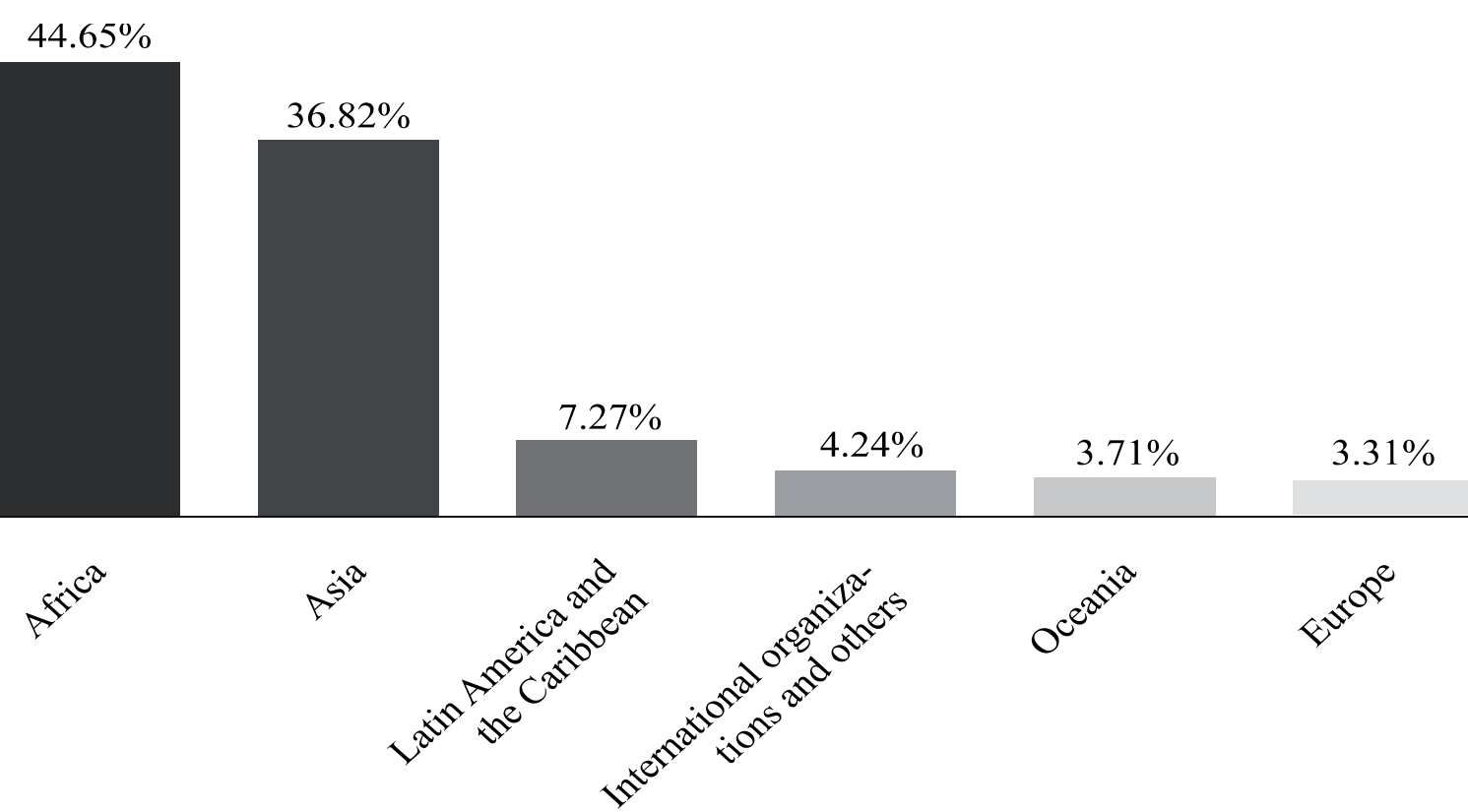

Fuente: El Consejo de Estado de la R. P. de China. http://english.www.gov.cn/archive/whitepaper/202101/10/content_WS5ffa6bbbc6d0f72576943922.html

El documento refleja los cambios habidos en la cooperación exterior china, tales como mayor inversión en los sectores de educación, salud y medio ambiente. Resalta también su apuesta multilateral con un apartado dedicado a la Agenda 2030 sobre Desarrollo Sostenible de Naciones Unidas, en el que apunta que "el primer objetivo de la Agenda 2020 es "acabar con la pobreza en todas sus formas y en

42 "Full text: China's International Development Cooperation in the new era", The State Council of the P.R. of China, 2021, disponible en http://english.www.gov.cn/archive/whitepaper/202101/10/ content_WS5ffa6bbbbc6d0f72576943922.html (consultado el 25/05/2021). 
todo el mundo"43. Además, reconoce como un "nuevo instrumento de ayuda" el Fondo de Asistencia para la Cooperación Sur-Sur, al que ha destinado desde su fundación en 2015 un total de 3.000 millones de dólares con los que ha puesto en marcha proyectos con numerosas organizaciones internacionales. Esto supone una diversificación de la ayuda que tradicionalmente se entregaba, en ocho formas: proyectos completos, bienes y materiales, cooperación técnica, cooperación en el desarrollo de recursos humanos, equipos médicos, voluntarios sobre el terreno, ayuda humanitaria de emergencia y alivio de la deuda ${ }^{44}$.

Otras destacadas novedades del tercer libro blanco son la referida a la igualdad de género y el impulso a la cooperación triangular. En línea con el reconocimiento de Mao Zedong de que "las mujeres sostienen la mitad del cielo", el documento indica que "las mujeres son una importante fuerza impulsora del progreso social" $^{\prime \prime 5}$ y asegura que China está redoblando sus esfuerzos para formarlas y empoderarlas en otros países en desarrollo. En cuanto a la cooperación tripartita se muestra favorable "como país en desarrollo" a buscar sinergias con los "países desarrollados y las organizaciones internacionales que tienen ventaja en capital y tecnología", siempre y cuando se respete plenamente "la soberanía y la voz" de los países receptores de la ayuda.

\section{Conclusión}

El presidente Xi Jinping, movido por la confianza política que inspiran los logros de la República Popular, quiere jugar la baza de la experiencia acumulada por China en su proceso de desarrollo para priorizar la lucha contra la pobreza desde la Agenda 2030 para el Desarrollo Sostenible de Naciones Unidas. Su apuesta por la paz y la seguridad, unida a un desarrollo innovador, coordinado, ecológico, abierto e inclusivo, revela la voluntad de China de abordar la última fase de su ascenso a la cúspide de la economía mundial como representante y voz de los países más desfavorecidos.

\footnotetext{
${ }^{43}$ lbídem...

${ }^{44}$ C. ZHANG Y Y. TANG (2021)

45 http://english.www.gov.cn/archive/whitepaper/202101/10/content_WS5ffa6bbbc6d0f72576943922.html (consultado el 25/05/2021).
} 
Ungido del Mandato del Cielo que le exige satisfacer las necesidades de su pueblo y proporcionarle una vida mejor, $X_{i}$ Jinping ha encontrado en la Franja y la Ruta la estrategia que confirma a la República Popular como actor fundamental en la economía regional y global. La iniciativa abre una serie de potencialidades económicas y tecnológicas, incluida la internacionalización del yuan, que consolidan el nexo entre China, Asia, África y el resto del mundo y que, con su visión a largo plazo, facilitan que China alcance 2049 convertida en un "país socialista moderno, próspero, fuerte, democrático, civilizado y armonioso". El presidente $X i$ asegura que ese es el "sueño chino" de cara al centenario de la fundación de la República Popular.

Pekín, con su enorme capacidad financiera que, a base de préstamos sin intereses, préstamos en condiciones favorables y subvenciones, se ha convertido en el primer prestamista de los países en vías de desarrollo, ha echado mano de la experiencia adquirida en décadas de cooperación con África, para ampliar su ayuda a otros países, bajo los principios de respeto a la soberanía y no injerencia en los asuntos internos. Su cooperación ha sido fundamentalmente bilateral, pero ahora está dispuesto a una triangulación con países industrializados y con organizaciones internacionales, que tienen una ventaja en capital y tecnología, para explorar juntos nuevas vías de desarrollo que mejoren el nivel de vida de los ciudadanos de los países receptores.

Sin embargo, los principios chinos para el desarrollo internacional no coinciden con los valores occidentales, lo que se ha traducido en modelos distintos de cooperación, cuya competitividad agrava las fricciones de la rivalidad desatada entre Estados Unidos y China. Los países en vías de desarrollo se encuentran en medio de este fuego cruzado y la mayoría se resiste a elegir entre una superpotencia o la otra.

Sin alianzas militares y con vecinos poco amigables, la ayuda exterior forma parte del ADN de la República Popular, que ve el desarrollo de los países pobres como una extensión del propio. Para China, el crecimiento económico es la panacea contra el descontento social, de ahí que su fórmula para hacer frente a los grandes retos mundiales sea paz y desarrollo, una estrategia útil para consolidar su dominio económico y construir "una comunidad de futuro compartido para la humanidad". 


\section{Bibliografía}

Berumen, S. A. (2009), "Evaluación de las reformas del Consenso de Washington en Brasil y México", disponible en http://revistas.bancomext.gob.mx/rce/magazines/130/3/704_Berumen.pdf

CARDenAl, J. P. y AraúJo, H. (2011), La silenciosa conquista china Una investigación por 25 países para descubrir como la potencia del siglo XXI está forjando su futura hegemonía, Barcelona, Crítica.

Cheng, C. (2019), "The logic behind China's Foreign Aid Agency", Carnegie Endowment for International Peace, disponible en https://carnegieendowment. org/2019/05/21/logic-behind-china-s-foreign-aid-agency-pub-79154

DIKÖTTER, F. (2017), La gran hambruna en la China de Mao. Historia de la catástrofe más devastadora de China (1958-1962), El Acantilado.

Fanjul, E. (2009), "El Consenso de Pekín: ¿ Un nuevo modelo para los países en desarrollo?", Real Instituto Elcano, disponible en http://www.realinstitutoelcano. org/wps/portal/rielcano_es/contenido?WCM_GLOBAL_CONTEXT=/elcano/ elcano_es/zonas_es/cooperacion+y+desarrollo/ari1 22-2009

FERCHEN, M. (2020), "How China is reshaping International Development", Carnegie Endowment for International Peace, disponible en https://carnegieendowment. org/2020/01/08/how-china-is-reshaping-international-development-pub-80703

FEWSMITH, J. (2004), "Promoting the scientific development concept", China leadership monitor, number 11, disponible en https://www.hoover.org/sites/default/files/ uploads/documents/clm 1 1_jf.pdf

GonZÁlEZ, C. F. (2021), El gran sueño de China Tecno-socialismo y capitalismo de Estado, Madrid, Tecnos.

Higueras, G. (2014), "Disidencia y Derechos Humanos en China", Revista del Instituto Español de Estudios Estratégicos, (3), disponible en https://revista.ieee. es/article/view/323

Hoffman, C. (1971), "The Maoist Economic Model", Journal of Economic Issues, vol. 5, n 3 (septiembre), 12-27, disponible en http://www.jstor.org/stable/4224090 
HuAng, Y. (2008), Capitalism with Chinese Characteristics Entrepreneurship and the State, Cambridge University Press.

KenNedY, S. (2010), "The Myth of Beiijing Consensus", Journal of Contemporary China, disponible en https://www.tandfonline.com/doi/pdf/10.1080/1067056 1003666087?needAccess=true

LAMPTON, D. M. (2008), The three faces of Chinese power, University of California Press.

LeE Myers, S. (2021), "An Alliance of Autocracies? China Wants to Lead a New World Order", NYT, 29/03/2021, disponible en https://www.nytimes. com/2021/03/29/world/asia/china-us-russia.html

Lumumba-Kasongo, T. (2011), "China-Africa relations: A Neo-Imperialism or a Neo-Colonialism? A reflection", African and Asian Studies, Brill, disponible en https://brill.com/view/journals/aas/10/2-3/article-p234_8.xml

MAÇAES, B. (2018), Beltand Road A Chinese world order, Londres, Hurst \& Company.

Quian, M. y Fremd, G. (2010), "El Consenso de Beijing", Letras Internacionales, disponible en https://revistas.ort.edu.uy/letras-internacionales/article/view/1206 $/ 1196$

Ramo, J. C. (2004), "The Beijing Consensus", The Foreign Policy Centre, disponible en https://fpc.org.uk/wp-content/uploads/2006/09/244.pdf

SPENCE, J. D. (2011), En busca de la China moderna, Barcelona, Tusquets.

Subramanian, A. (2013), Eclipse A la sombra del dominio económico chino, Barcelona, Destino.

Tamames, R. (2007), El siglo de China De Mao a primera potencia mundial, Barcelona, Planeta.

TANG, R., "China's New Development Opens Up New Opportunities for the World. Embassy of the People Republic of China in Malaysia, disponible en http://my.chinaembassy.org/eng/sgxw/†1840912.htm (consultado el 03/05/2021).

Wheatley, J., CotterilL, J. y MunshI, N. (2020), "African debt to China: 'A major drain on the poorest countries'", Financial Times, disponible en https://www. ft.com/content/bd73al 15-1988-43aa-8b2b-40a449da 1235 
XI, J. (2014), La gobernación y la administración de China, Pekín, Ediciones en Lengua Extranjera.

YANG, J. (2011), "Rethinking the Beijing Consensus: how China respond to crises", The Pacific Review, vol. 24, $\mathrm{n}^{\circ} 3$ (tandfonline.com).

YANG, J. (2015), "China's new diplomacy under Xi Jinping Administration", China Quarterly of International Strategic Studies, vol. 1, $\mathrm{n}^{\circ} 1$ 1, 1-17, disponible en https:// www.worldscientific.com/doi/10.1142/S2377740015500013

YAO, Y. (2010), "The end of the Beijing Consensus Can China's model of authoritarian growth survive?", Foreign Affairs, 2/02/2010, disponible en hitps://www. foreignaffairs.com/articles/china/2010-02-02/end-beijing-consensus

ZhANG, C. y TANG, Y. (2021), "What does a new white paper tell us about China's international aid?", The Diplomat, 13/01/2021, disponible en https:// thediplomat.com/2021/01/what-does-a-new-white-paper-tell-us-about-chinas -international-aid/

ZHANG, D., "Messages from China's third white paper on foreign aid", The Interpreter, Lowy Institute, disponible en https://www.lowyinstitute.org/the-interpreter/ messages-china-s-third-white-paper-foreign-aid

ZhANG, D. y J, H. (2020), "The new Chinese aid agency after its first two years", disponible en https://devpolicy.org/the-new-chinese-aid-agency-after-its-first-twoyears -20200422-2/

ZhANG, D. y TRABber WAISBICH, L. (2020), "Trilateral development cooperation: the cases of China anda Brazil", disponible en https://devpolicy.org/trilateral-development -cooperation-the-cases-of-china-and-brazil-20200707-2/

ZHANG, H. (2021), "China's manifesto for leadership in global development", disponible en https://pandapawdragonclaw.blog/2021/02/08/chinas-manifesto -for-leadership-in-global-development/

"Primer Libro Blanco: Desarrollo Pacífico de China", Ministerio de Asuntos Exteriores de la R. P. China, disponible en https://www.fmprc.gov.cn/esp/zxxx/\$862206. shtml (consultado el 31/05/2021)

"Diez planes quinquenales de desarrollo económico y social de la Nueva China", IV Sesión de laXAPN, disponible en http://spanish.china.org.cn/spanish/222422.htm 
"China publica libro blanco sobre ayuda al extranjero", Diario del Pueblo, 10/07/2014, disponible en http://spanish.peopledaily.com.cn/n/2014/0710/ c31621-8753691.html (consultado el 6/06/2021)

"Full text: China's International Development Cooperation in the new era", The State Council of the P.R. of China, 2021, disponible en http://english.www.gov.cn/ archive/whitepaper/202101/10/content_WS5ffa6bbbc6d0f72576943922.html

Segunda Conferencia de Alto Nivel de Naciones Unidas sobre Cooperación Sur-Sur, disponible en https://www.un.org/pga/73/es/2019/03/21/ second-high-level-united-nations-conference-on-south-south-cooperation-2/

"China emite libro blanco sobre cooperación internacional para el desarrollo", Xinhua, disponible en http://spanish.xinhuanet.com/2021-01/10/c_139655778.htm

"China's International Development Cooperation in the New Era", The State Council Information Office, PRC, 2021, disponible en http://english.scio.gov.cn/whitepapers/2021-01/10/content_77099782_8.htm

" ¿Conoces la Nueva Agencia de Cooperación Internacional para el desarrollo de China? Disponible en https://www.actuemosjuntos.org/post/conoces-la-nuevaagencia-de-cooperaci\%C3\%B3n-internacional-para-el-desarrollo-de-china 